\title{
A nova definição do quilograma em termos da constante de Planck
}

The new definition of the kilogram in terms of Planck's constant

\author{
Luiz Paulo Damaceno ${ }^{1}$, Richard Mascarin ${ }^{1}$, João Marcelo Pereira Nogueira ${ }^{1}$, Daniel Varela \\ Magalhães $^{2}$, Vanderlei Salvador Bagnato*1@ \\ ${ }^{1}$ Universidade de São Paulo, Instituto de Física de São Carlos, São Carlos, SP, Brasil \\ ${ }^{2}$ Universidade de São Paulo, Escola de Engenharia de São Carlos, São Carlos, SP, Brasil
}

Recebido em 12 de Outubro, 2018. Aceito em 20 de Novembro, 2018.

\begin{abstract}
A partir de 2019, a unidade de massa do Sistema Internacional de Unidades (SI), o quilograma, terá sua definição modificada. Deixará de ser o artefato metálico guardado na sede do BIPM, nos arredores de Paris, para ser baseado na constante de Planck (h). Qual é a necessidade desta mudança? E como as medidas são realizadas? São algumas das perguntas que respondemos nesta comunicação, onde descrevemos a operação do experimento denominado Balança de Watt utilizado para a nova maneira de realizar a unidade de massa. Também detalhamos como montamos uma Balança para fins didáticos, utilizada em apresentações sobre o tema. O tópico é de interesse de todos, mas em especial para alunos que recebem aulas de física em qualquer uma das áreas de formação.

Palavras-chave: quilograma, balança de Watt, novo SI.
\end{abstract}

In 2019, the unity of mass of the International System of Units (SI), the kilogram, will have its definition modified. It will not be anymore the metallic artifact kept in the BIPM building, in the neighborhood of Paris, to be based on Planck's constant (h). What is the need for this change? And how the measurements will be done? These are some of the questions that will be answered at this communication, where we describe the operation of the experiment named Watt Balance used in the new way to realize the unity of mass. Besides that, we will describe how we assembled a Balance for teaching purposes, used in talks about the theme. The topic interests everybody, but in special students that take physics courses in any area.

Keywords: kilogram, Watt balance, new SI.

\section{Introdução}

Realizar uma medida é fazer uma comparação. Para que a medida de algo possa ser entendida por todos é preciso que tenhamos um referencial para comparação. Esta é a origem de um sistema de unidades de uso internacional. Ao medirmos tempo todos utilizam o segundo (s), ao medirmos comprimento todos utilizam o metro $(\mathrm{m})$ e ao medirmos massa todos usam o quilograma (kg) desde que o Sistema Internacional de Unidades foi criado [1]. Ao usarmos tais convenções é preciso que todas estas unidades sejam bem definidas. A história de como tudo isto foi feito remonta a vários séculos e precisaria de muitas páginas para poder descrevê-la. Esta descrição histórica é rica e importante mas, apesar disto, iremos deixá-la para ser discutida por especialistas [2]. Aqui vamos nos ater à história mais recente e mesmo aos fatos que acontecerão publicamente apenas em 2019 [3].

Ao definirmos nosso padrão de unidade temos que ser cuidadosos para que ele obedeça a certos requisitos básicos. Primeiro um padrão não pode sofrer grandes variações, afinal ele é a referência e, quando sofre qual-

*Endereço de correspondência: vander@ifsc.usp.br. quer alteração, todo o resto é afetado. No passado o comprimento era medido pelo tamanho do pé do rei. Este é um padrão terrível, já que não apenas o tamanho do pé depende da idade, mas também cada rei tem um pé de tamanho diferente. O comprimento de um objeto, ora medido e documentado, poderia não mais servir quando viesse um novo rei. No momento em que isto tornou-se problemático foi necessário definir a unidade de comprimento de uma forma mais constante na natureza, deixando então de ser baseado no corpo humano e passando a ser baseado no planeta Terra. No meio disto, foi criado o sistema métrico (com grande participação do famoso matemático Gauss em 1852) [4].

Com ele, o metro e o quilograma passaram a ser definido com base em artefatos mantidos de forma segura nas proximidades de Paris - França [5]. Enquanto o metro foi definido como sendo a distância entre duas marcas de uma barra de uma liga de platina e irídio, o quilograma foi definido como a massa contida num bloco da mesma liga. Ambos artefatos ficaram sob custódia do BIPM ( $B u$ reau International des Poids et Mesures). Muitas cópias deste padrão foram feitas e distribuídas por todos os países signatários da Convenção do Metro. Junto com metro 
e quilograma vieram muitas outras unidades essenciais para qualificação de grandezas fundamentais. Enquanto na Figura 1 mostramos os artefatos metálicos para metro e quilograma, na Figura 2 mostra-se a confecção de unidades básicas do Sistema Internacional de Unidades, como estabelecido atualmente.

Há certamente, muito mais unidades que utilizamos no dia-a-dia do que as especificadas na Figura 2. No entanto todas podem ser obtidas destas sete Unidades de Base. As outras unidades são denominadas de Unidades Derivadas.

\section{O Problema com os Artefatos}

O sistema métrico de unidades e o estabelecimento de padrões internacionais trouxe um enorme progresso para o homem. Passamos a fazer medidas que poderiam ser feitas em épocas separadas e mesmo assim comparadas. O Sistema Internacional com unidades baseadas em artefatos certamente foi um grande avanço. Ocorre, no entanto, que estes artefatos não são imutáveis. De fato, medidas periodicamente realizadas entre artefatos que usam cópias idênticas no começo do século XX mostram grandes variações [6]. Isso mostrou que padrões e definições baseadas em artefatos também são frágeis.

A fim de alterar esta situação, físicos começaram a buscar formas de definição baseada em valores que não se alteram no tempo. Os melhores candidatos para isso são

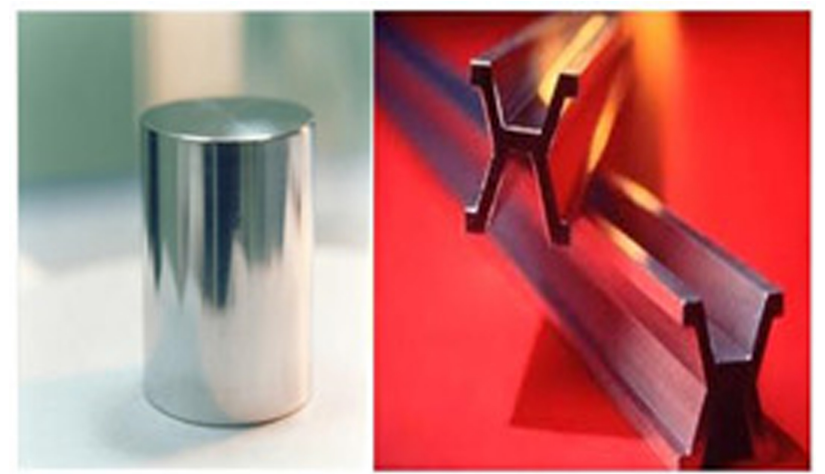

Figura 1: Artefatos que definiram o quilograma e o metro. [Fonte: www.wikipedia.org]

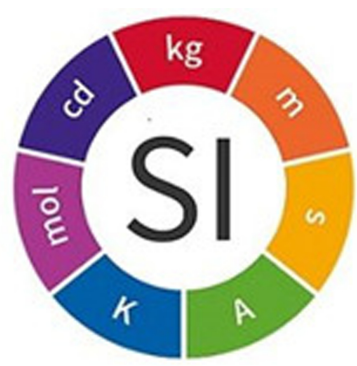

Figura 2: Novo Sistema Internacional definidas composto por sete unidades. Há na verdade muitas outras definidas a partir destas. [Fonte: www.bipm.org] as constantes fundamentais, pois são valores associados a fenômenos naturais que nunca se modificam, sendo por isto observadas como constantes.

A primeira das unidades do SI a passar por uma adequação foi o segundo. Inicialmente baseado nos movimentos de rotação e translação da Terra, o segundo passou a ser baseado na frequência da radiação eletromagnética necessária para efetuar eficientemente a transição entre os estados hiperfinos do átomo de Césio-133. O átomo de Césio não muda com o tempo e, portanto, a transição entre seus estados eletrônicos é uma constante. A chamada definição atômica do tempo teve sua realização consagrada com os Relógios Atômicos [7]. Com o tempo, passamos a buscar novas definições para as demais unidades do SI.

Em seguida veio a definição do metro, que deixou de ser a distância entre duas marcas do padrão mantido na França. Inicialmente foi definido com base no comprimento de onda da transição do átomo de Criptônio para, finalmente, ser definido em termos da velocidade da luz no vácuo [1]. Toda radiação eletromagnética, propagase no vácuo com a mesma velocidade $(\mathrm{c}=299.792 .458$ $\mathrm{m} / \mathrm{s}$ ). A velocidade da luz no vácuo é uma das grandezas mais constantes da natureza e base para muitos efeitos. Se já sabemos medir tempo tão bem, podemos usar o tempo aliado à velocidade da luz para definir que $1 \mathrm{~m}$ é a distância percorrida pela luz no vácuo no tempo $\mathrm{t}$ $=1 / 299.792 .458$ segundos. Nas últimas décadas, tempo (segundo) foi definido na constante natural associada ao átomo de Césio e o comprimento (metro) foi definido baseado na constante da velocidade da luz no vácuo. Como podemos tomar o mesmo caminho com a massa $(\mathrm{kg})$ ? Esta continua sendo baseada no artefato mantido sob sete chaves ao sul de Paris.

\section{A Nova Definição do kg}

Se formos utilizar a mesma filosofia praticada na definição do segundo e do metro, baseado em constantes fundamentais da natureza, qual seria esta constante? Após muitas considerações e debates, a constante escolhida para ser a base na definição do $\mathrm{kg}$ foi a constante de Planck (h). A constante de Planck $\left(\mathrm{h}=6,62 \times 10^{-34} \mathrm{~m}^{2} \mathrm{~kg} / \mathrm{s}\right)$ aparece na natureza pela primeira vez na explicação da radiação de um corpo aquecido [8] pelo próprio Planck e acaba sendo a quantidade fundamental na formação da mecânica quântica.

Antes de continuarmos a explicação de como é possível definir o $\mathrm{kg}$ à partir da constante de Planck é preciso esclarecermos alguns pontos importantes.

Primeiro, é importante entender a filosofia por trás da definição de uma unidade com base em uma constante natural. A Figura 3 ilustra essa filosofia.

Utilizando o padrões existentes, determinamos da melhor forma possível a constante. Isto faz com que percorramos o caminho 1 do diagrama da Figura 3. Uma vez que a constante $\mathrm{h}$ é determinada no melhor nível 


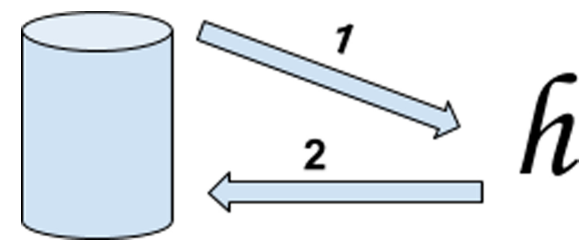

Figura 3: Definindo uma unidade de medida baseando-se em uma constante natural.

possível, ela passa então a ser definida como tendo um valor exato. No passo seguinte, usando a definição com este valor exato, tomamos agora o caminho $\mathbf{2}$ e passamos a determinar a unidade a partir da constante fundamental. O segundo ponto importante além da definição é a realização da unidade de medida. Esta parte é muito importante pois é a operação que permite, de fato, disponibilizar a unidade de medida de forma irrestrita para seu uso. No caso do kg esta realização é feita através da chamada balança de Watt ou Kibble [9]. Ao explicarmos como a balança funciona estaremos, ao mesmo tempo, demonstrando como é a conexão do quilograma com a constante h.

\section{A Balança de Watt e a Realização do $\mathrm{kg}$}

Vamos explicar os princípios e definições para entendermos como o quilograma pode ser realizado usando a balança de Watt, considerada a forma mais adequada para isto. Vamos começar considerando a situação onde um campo magnético de amplitude B e direção alinhada ao eixo de uma bobina, como indicado na Figura 4, vai interagir com o circuito desta.

Inicialmente, como o campo magnético não é conhecido, provocamos a interação deste campo com um circuito formado por uma bobina de lado L, deslocando-se com velocidade $v$ transversal ao sentido do campo. Ao des-

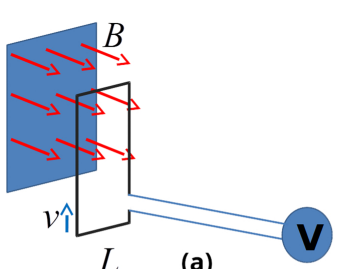

(a)

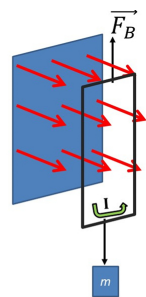

(c)

$$
\begin{aligned}
& F_{B}=I L B \\
& F_{B}=P=m g \\
& m g=I L B \\
& L B=\frac{V}{v}
\end{aligned}
$$

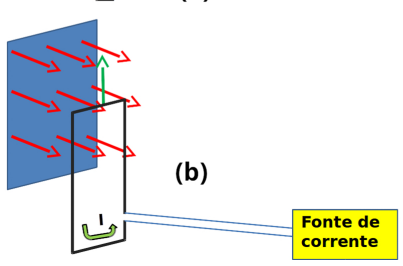

Figura 4: Princípio da balança de Watt: (a) Campo e geometria definidos pela indução e velocidade; (b) A força magnética agindo com a aplicação de corrente; (c) Equilíbrio de forças. locarmos esta bobina no campo, temos que a tensão induzida $(V)$ pode ser obtida utilizando a Lei de Indução de Faraday [10]:

$$
V=L B v
$$

Neste caso, uma vez conhecidas as características geométricas da bobina $(L)$, o campo $(B)$ pode ser obtido no seu produto através do conhecimento da tensão induzida $V$ e da velocidade $v$, pela relação:

$$
L B=V / v
$$

Esta é uma operação que permite a determinação de uma quantidade que servirá de referência a dois parâmetros importantes: $L$ e $B$. O conhecimento de $V$ e $v$, permite a determinação de $L B$. Com a mesma bobina e o mesmo campo invertemos o seu uso e fazemos circular uma corrente elétrica I. Como resultado desta corrente surge na bobina uma força magnética, que pela Lei do Eletromagnetismo [11] vale:

$$
F_{B}=I L B
$$

Esta situação está indicada na Figura 4b.

Se colocarmos acoplada nesta bobina uma massa $\mathrm{m}$, como indicado na Figura - 4c, podemos regular a corrente $I$ até que a força $\mathrm{F}_{\mathrm{B}}$ equilibre o peso da massa $m$, de valor $m g$. Nesta situação:

$$
F_{B}=m g
$$

Finalmente,

$$
m g=I L B
$$

Como $L B=V / v$, também obtido na fase indicada na Figura $4 \mathrm{a}, m * g=I *(V / v)$ :

$$
m g v=I V
$$

Na equação acima, o equilíbrio é estabelecido quando a potência mecânica $(m g v)$, iguala-se à potência elétrica $(V I)$. Ambos têm como unidade o watt e por isto, esta balança que equilibra potências é chamada "Balança de Watt". O interessante na equação acima é que, conhecendo a aceleração da gravidade $(g)$, a velocidade $(v)$, a corrente para equilibrar a massa $(I)$ e a tensão induzida obtida no momento da calibração $(V)$, podemos determinar a massa $(m)$. Se estabelecermos que todas grandezas tem que ser tal que $m=1 \mathrm{~kg}$, este experimento torna-se a realização do quilograma. Para que isto possa ser feito com exatidão é preciso determinar muito bem a tensão elétrica $(V)$, a corrente $(I)$, a velocidade $(v)$ na fase de calibração e a aceleração da gravidade no local.

Para medirmos a tensão elétrica com precisão, usamos a definição do padrão de tensão através do Efeito Josephson Alternado [12]. Este efeito está esquematizado na Figura 5. Em linhas gerais, passando uma corrente alternada numa junção composta de dois supercondutores e uma fina camada isolante, uma tensão :

$$
V=f(h /(2 e))
$$




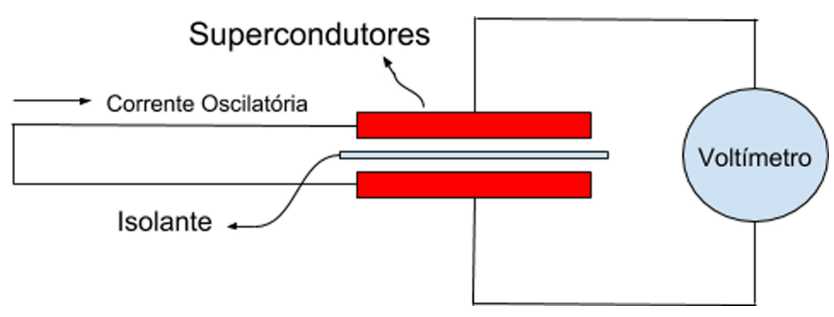

Figura 5: Medição da tensão através da junção Josephson, na qual aplica-se uma corrente constante e micro-ondas no material isolante.

aparece na junção. A tensão depende da frequência da corrente alternada ( $f$ e das constantes de Planck $(h$ e da carga do elétron $(e)$. De fato, como a frequência ( $f$ pode ser medida com altíssima resolução, o conhecimento de $h$ e $e$ permite medir $V$ com grande confiabilidade. Para medirmos uma tensão $V$ qualquer, como a da expressão acima, podemos usar uma pilha de junções, com $n$ junções tal que:

$$
V=n f(h /(2 e))
$$

Desta forma, a tensão será medida através das junções de Josephson, que dependem diretamente da constante $h$.

Para medir a corrente, usaremos uma resistência padrão, obtida através de outro efeito quântico importante, o chamado efeito Hall quântico. Nele o padrão de resistência elétrica $R=(1 / p)\left(h / e^{2}\right)$ com $p$ um número inteiro.

Usando a lei de Ohm, e o esquema da Figura $6, I=$ $V^{\prime} / R$, onde $V^{\prime}$ é uma nova tensão usada para determinar a corrente. Novamente, $V^{\prime}=n^{\prime} f(h /(2 e))$, de modo que:

$$
I=n^{\prime} p f(e / 2)
$$

no qual $n^{\prime}$ é um novo número inteiro. Desta forma, combinamos $V$ e $I$ na expressão que determina a massa.

$$
m g v=\left(h f^{2}\right) /\left(4 p n n^{\prime}\right)
$$

ou finalmente:

$$
m=\left(\left(f^{2}\right) /\left(4 p n n^{\prime} g v\right)\right) h
$$

Definindo a constante de Planck $h$ e medindo $f p n n^{\prime}, g v$ determinamos $m=1 \mathrm{~kg}$, estando portanto definida a massa.

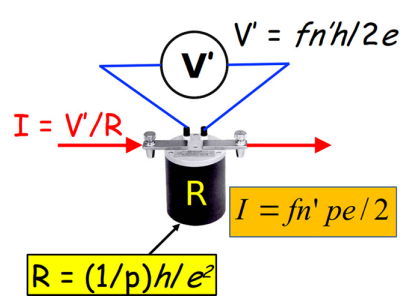

Figura 6: Efeito Hall quântico para medida de corrente.

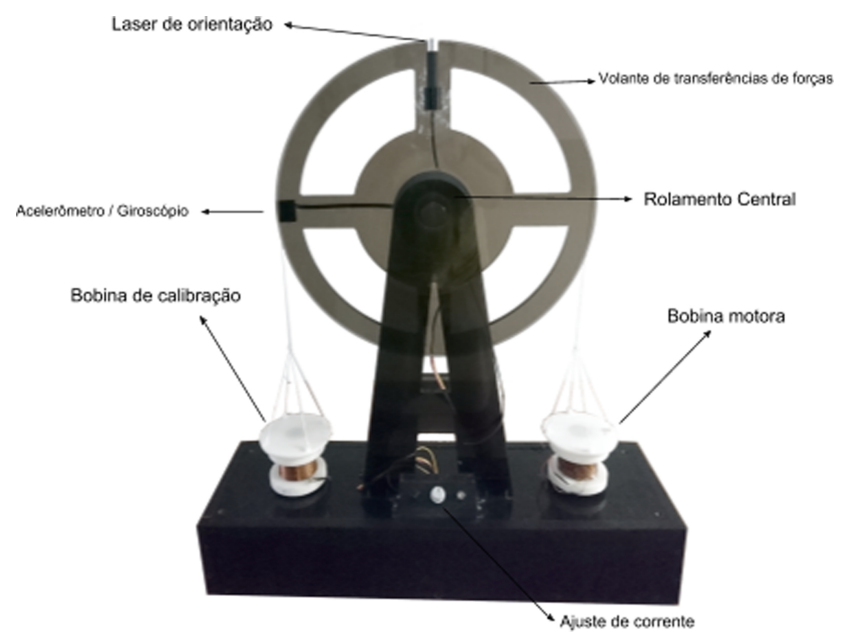

Figura 7: Balança de Watt construída para fins didáticos.

Todo este princípio que discutimos é posto em prática através da balança mostrada na Figura 7 no qual duas bobinas, cujo comprimento de fio L não é conhecido. Estas bobinas estão inseridas no campo de um ímã permanente $B$, que também não é conhecido. Um modo de operação de calibração vai permitir definir $B L$. Neste caso, uma das bobinas é escolhida como padrão e a outra usada para fazer o sistema se deslocar enquanto mede-se $V$ e $v$. A velocidade pode ser obtida com medidas precisas de deslocamento e tempo atraveś de interferometria óptica. Finalmente temos que adicionar massa mantendo todo resto fixo, até atingirmos o valor de $1 \mathrm{~kg}$. A balança permite realizar o $\mathrm{kg}$ com precisão relativa de $10^{-8}$, muito melhor do que qualquer artefato que poderíamos produzir.

\section{A balança de Watt construída e seus detalhes}

Na configuração da balança foram utilizados um volante circular usinado em acrílico com $0,2 \mathrm{~m}$ de raio, fixado em um rolamento e sustentando por um suporte vertical de 0,4 $\mathrm{m}$ de altura, permitindo girar em ambos os sentidos conforme mostra a Figura 7.

Duas bobinas foram construídas, enroladas com 750 espiras de fio de cobre esmaltado com seção de 0,001 $\mathrm{mm}^{2}$ em um carretel impresso em ABS. Sobre o carretel temos uma base circular de 0,08 m de diâmetro, onde poderemos posicionar as diferentes massas utilizadas durante as medidas. As bobinas foram fixadas de modo que ficassem suspensas no ar, contra balanceadas nas laterais do volante, conforme mostrado na Figura 7. Quando o volante se move as bobinas se movimentam como consequência, encaixando-se em pequenas hastes de fixação onde estão localizados os ímãs de neodímio, ferro e boro do tipo Nd2Fe14B. A função do ímã é prover o campo magnético que corta as espiras da bobina, Figura 8. 

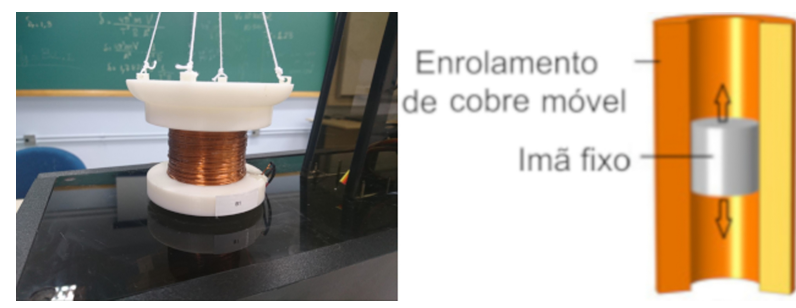

Figura 8: Bobina com 750 espiras com a disposição do ímã fixo, a bobina possui movimento livre sobre um eixo vertical (haste) que fixa o ímã.

Foi desenvolvido um sistema eletrônico microcontrolado que interage diretamente com as bobinas, de modo a fazer leitura de parâmetros e atuar para fazer as medidas.

Na bobina de calibração será medida a tensão induzida no momento calibração. Na bobina motora serão aplicadas a corrente durante a medida da massa (com possibilidade de ajuste manual) e a corrente variável para oscilar mecanicamente a bobina durante a etapa de calibração. Nesse caso, as duas bobinas possuem o mesmo valor $B L$ (idênticas). Assim podemos utilizar qualquer uma delas como bobina de calibração e bobina motora.

No controle da balança um microcontrolador do tipo Arduino MEGA, mostrado na Figura 9, processa os dados coletados referentes a posição, velocidade, corrente, tensão induzida, aceleração da gravidade e massa. No fim da medida os dados processados são transferidos serialmente para um computador. Para deixar o sistema independente de computadores externos, foi utilizado um computador embarcado do tipo Raspberry PI, mostrado na Figura 10, no qual podemos exibir os dados processados pelo Arduino em uma saída de vídeo do tipo HDMI (High-Definition Multimedia Interface) com a ajuda de um pequeno programa em formato de script.

No sistema de apoio para coleta de dados, foram selecionados sensores de corrente (feito com um amplificador operacional e um resistor de referência) e um sensor de posicionamento constituído de um giroscópio e um acelerômetro (MPU6050).

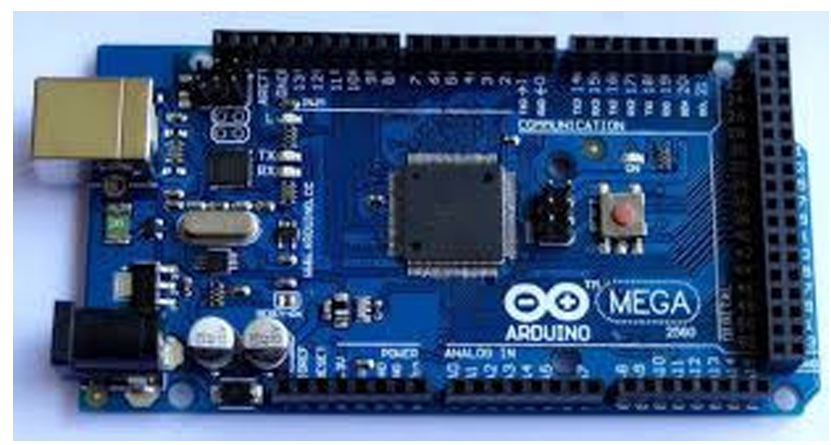

Figura 9: Plataforma Arduíno, modelo MEGA 2560, utilizado na aquisição e no processamento dos valores de corrente, tensão, velocidade, massa final e características magnéticas das bobinas.

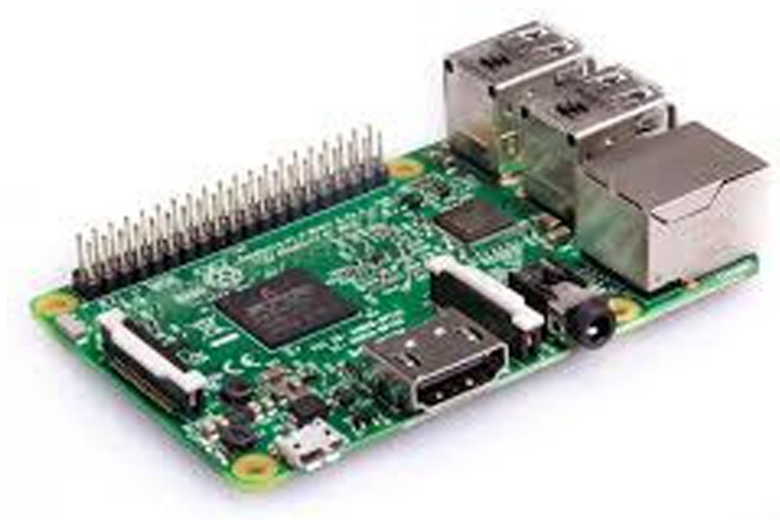

Figura 10: Raspberry Pi 3 utilizado como computador de visualização dos dados, o Arduíno comunica-se com este embarcado via comunicação serial.

Na medida da corrente que é aplicada à bobina motora foi usado um resistor de referência, de $1 \Omega / 50 \mathrm{~W} 5 \%$ com baixo coeficiente de variação térmica $\left( \pm 50 \mathrm{ppm} /{ }^{\circ} \mathrm{C}\right)$ modelo WH50-1R0 e um amplificador operacional do tipo CA3140, alimentado com fonte simples de $12 \mathrm{~V}$ (Figura 11). A configuração selecionada é do tipo não-inversora, com ganho aproximado de 21 ao valor de tensão obtido na resistência conectada em série com bobina.

Na medida da bobina de calibração utilizamos apenas o estágio de ganho do amplificador não sendo necessário o uso do resistor de referência.

Conhecendo a posição e a excursão das bobinas podemos criar uma oscilação e medir a velocidade de movimentação da balança. Dessa forma conseguimos realizar a caracterização com o auxílio de um sensor composto

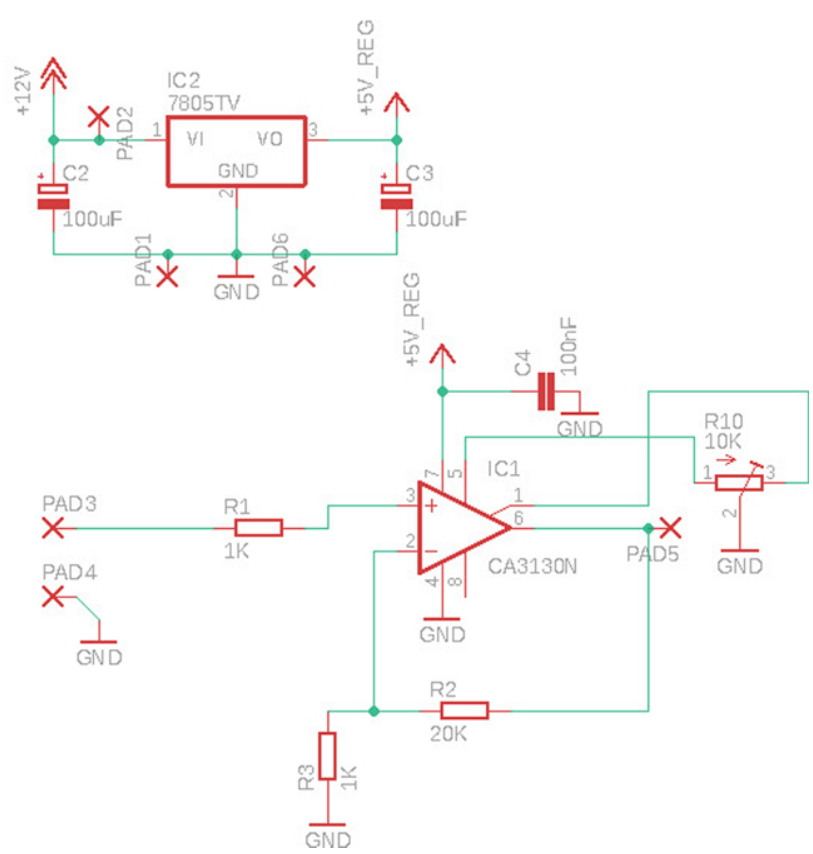

Figura 11: Circuito montado para amplificar os sinais de corrente e de tensão induzida. 
de um acelerômetro e de um giroscópio. O acelerômetro é usado para estimarmos a aceleração da gravidade do local em que a balança se encontra e o giroscópio para sabermos a velocidade da bobina. O sensor escolhido para essa tarefa é o MPU6050 mostrado na Figura 12.

O controle de corrente é aplicado apenas na bobina motora e é realizado por modulação de largura de pulso, PWM (do inglês Pulse Width Modulation). Pode-se então controlar a corrente que circula pela bobina por meio de um circuito interface de saída. O circuito integrado L298 mostrado na Figura 13 possui uma ponte-H, onde 4 transistores funcionam anti-simetricamente como chaves, permitindo a circulação da corrente em ambos os sentidos pela bobina. $\mathrm{O}$ integrado permite operar com potência de até $25 \mathrm{~W}$, fornecendo corrente de pico de até $3 \mathrm{~A}$.

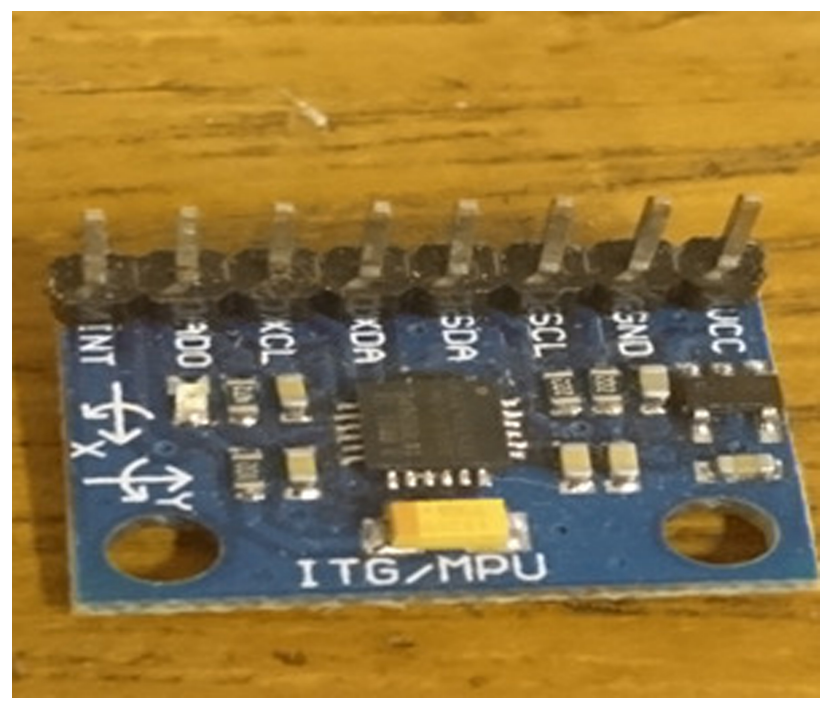

Figura 12: MPU6050, Giroscópio / Acelerômetro.

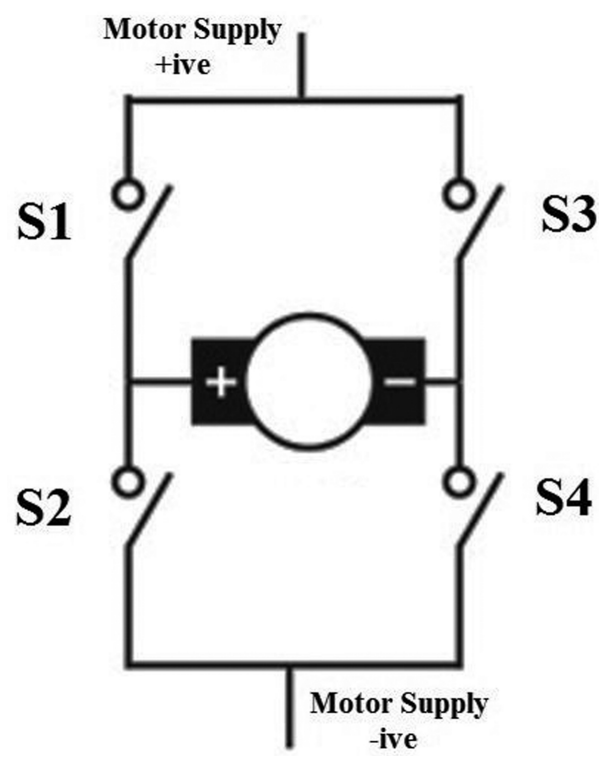

Figura 13: Ponte-H, com o integrado L298 para controle de corrente da bobina motora.
O sistema eletrônico é conectado a uma fonte de alimentação de $24 \mathrm{~V}$ x $3 \mathrm{~A}$, responsável pela alimentação do circuito de controle e pela corrente que circula nas bobinas durante a medição da balança. Fontes lineares nessa aplicação são mais interessantes do que o uso de fontes chaveadas pois produzem menos ruídos, originados por sinais harmônicos produzidos durante o chaveamento da fonte.

No projeto também foram utilizados circuito reguladores de tensão para gerar sinais com níveis adequados e com maior estabilidade nas linhas de alimentação do controlador e os os circuitos que compõem a balança.

\section{Modo de Operação}

Na operação da balança, foram programadas duas etapas no controlador, etapa de calibração e etapa de medição.

Durante a etapa de calibração o controlador recebe dados de posição máxima, média e mínima das bobinas, para que esses extremos possam ser aplicados no momento da oscilação. As posições são ajustadas manualmente pelo operador, posicionando as bobinas para cima e para baixo e pressionando um botão para armazenar os dados para cada posição.

Com os limites definidos o programa inicia a oscilação, fazendo circular corrente pela bobina motora. As bobinas se movimentam para cima e para baixo, conforme o sentido da corrente é alternado, numa velocidade constante que é medida e conferida pelo sensor de velocidade. A tensão induzida na bobina de calibração é também medida e guardada em memória, para o cálculo da relação de campo magnético e comprimento da bobina $(B L)$ que deve ser dada com $\mathrm{V} / \mathrm{m} / \mathrm{s}$. Esses valores são usados posteriormente pelo sistema no cálculo das massas durante etapa de medição.

No nosso sistema uma caracterização prévia das bobinas foi realizada e $B L$ foi medido em $5,65 \mathrm{~V} / \mathrm{m} / \mathrm{s}$. Esse valor é então armazenado como uma constante de referência na memória do controlador. Na etapa de calibração de medida o sistema deve oscilar por um tempo até que igualamos $B L$ a $5,65 \mathrm{~V} / \mathrm{m} / \mathrm{s}$.

Na etapa final de medição o operador coloca a massa de interesse sobre uma das bobinas, desequilibrando a balança. A seguir deve girar um potenciômetro para ajustar manualmente a corrente necessária para equilibrar a massa que deseja ser medida no sistema à força magnética gerada na bobina motora. Para auxiliar nessa tarefa um LED é acionado quando a posição de equilíbrio é atingida.

Ao chegar na posição de equilíbrio o programa confere o valor de aceleração da gravidade (medida em tempo real pelo acelerômetro no momento da medida de massa) e o valor de corrente que circula na bobina motora, fazendo o cálculo do valor de massa.

Durante as medidas realizadas no laboratório, foi possível aferir diferentes medidas para massas, dentro da faixa de 50 a 130 gramas. 


\section{Programação}

Na figura 14, um fluxograma representa o que está programado no Arduíno, além de um link com um repositório em que se encontra o código fonte com todo o programa construído para controlar a balança, é apresentado. Todos os pinos utilizados e que devem ser conectados estão declarados na função void setup().

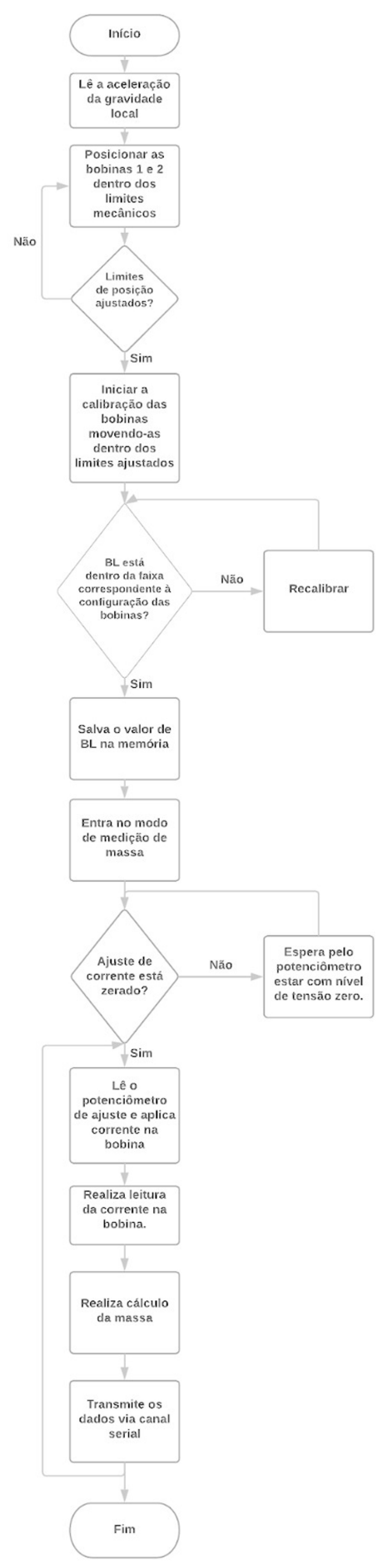

Figura 14: Fluxograma correspondente à programação presente no Arduíno Mega.
Link para o código fonte e esquemáticos (será atualizado conforme novas modificações forem feitas): https: //github.com/LuizPauloDamaceno/watt_ballance

\section{Conclusão}

A montagem e o funcionamento da Balança de Watt, mesmo para fins didáticos, não é tão trivial quanto parece. Além de toda a montagem eletrônica foi necessário realizar melhorias no sistema mecânico para que o mesmo sofra o mínimo possível com qualquer tipo de inércia e histerese.

Quando se lida com histerese, existe um fenômeno interessante que ocorre enquanto ao tentar equilibrar uma massa: As bobinas e o disco começam a mover-se somente depois que uma certa "quantidade" de força é aplicada, o que determina uma leitura errônea enquanto a corrente é incrementada para que a massa seja equilibrada.

O projeto mostrado no artigo refere-se a uma balança que consegue equilibrar até 135 gramas de massa e é utilizada para fins didáticos de ensino. Para massas maiores são necessários redimensionamentos nas bobinas, principalmente. Além disso, seriam necessárias alterações nos circuitos de condicionamento de sinal de corrente e algumas mudanças estruturais. Ainda assim, para $1 \mathrm{~kg}$, $10 \mathrm{~kg}, 100 \mathrm{~g}$ ou $1 \mathrm{~g}$ o princípio utilizado é o mesmo.

A Balança de Watt faz com que não precisemos mais de uma "referência preciosa". Agora trabalhamos somente com constantes fundamentais, abrindo a possibilidade de termos, cada um da sua forma e montando com o desenho que preferirmos, uma Balança de Watt. Cada um pode ter um meio de garantir que $1 \mathrm{~kg}$ seja realmente $1 \mathrm{~kg}$ como definido pelo SI. Nossa balança exibida opera de forma manual, mas futuramente com novas melhorias mecânicas e eletrônicas temos a pretensão de implementar um sistema de controle inteligente, que fará a variação de corrente necessária na bobina de acordo com o deslocamento sentido por um sistema de medida de posição angular.

\section{Agradecimentos}

Todos os participantes deste trabalho são membros do Centro de Pesquisa em Óptica e Fotônica / CEPID, e agradecem ao apoio da FAPESP - Projeto 2013/07276-1, CNPq e CAPES. Luiz Paulo Damaceno e Richard Mascarin são alunos do curso de Tecnologia em Manutenção de Aeronaves do Instituto Federal de São Paulo.

\section{Referências}

[1] BUREAU INTERNATIONAL DES POIDS ET MESURES, Sistema Internacional de Unidades - SI (Instituto Nacional de Metrologia, Normalização e Qualidade Industrial - INMETRO, Rio de Janeiro, 2012), p. 94.

[2] N.I. Brasil, Sistema Internacional de Unidades (Interciência, Rio de Janeiro, 2002). 
[3] T. Quinn, From Artefacts to Atoms: The BIPM and the search for ultimate measurement standards (Oxford University Press, New York, 2018), p. 440.

[4] W. Hallock e H.T. Wade, Outlines of the evolution of weights and measures and the metric system. (The Macmillan Co. Londres, 1906), p. 200.

[5] BUREAU INTERNATIONAL DES POIDS ET MESURES, History of the Pavillon de Breteuil, disponível em https://www .bipm.org/en/about-us/pavillonde-breteuil/, acessado em 24/11/2018.

[6] M. Stock, P. Barat, R.S. Davis, A. Picard e M.J.T. Milton, Metrologia 52, 310 (2015).

[7] J. Jespersen e J. Fitz-Randolph, From Sundials to Atomic Clocks: Understanding Time and Frequency (National Institute of Standards and Technology, Washington, 2011), p. 304.

[8] B. Toman, J. Fischer e C. Elster, Metrologia 49, 567 (2012).

[9] I.A. Robinson e S. Schlamminger, Metrologia 53, A46 (2016).

[10] M.N.O. Sadiku, Elements of Electromagnetics (Nova Iorque: Oxford University Press, 2007), $4^{\mathrm{a}}$ ed.

[11] http://physics.bu.edu/ duffy/PY106/ Electricgenerators.html

[12] B.D. Josephson, Phys. Lett. 1, 251 (1962). 